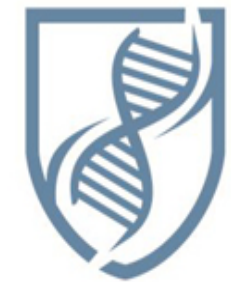

Journal of Bioscience and Applied Research

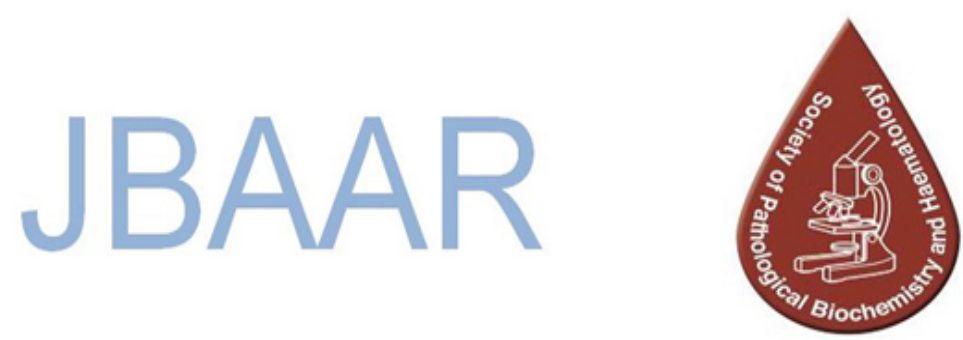

WWW.JBAAR.ORG

\title{
Neurobehavioral disorders in a group of children suffering from idiopathic epilepsy
}

\author{
*Shewikar El Bakry;**Soha Abdel Hady Ibrahim ; Hasnaa Abdelrhman Abdallah \\ *Psychiatry department, **Pediatrics department Faculty of Medicine, Benha University
}

(Corresponding author-email: d.hasnaa2@yahoo.com)

\begin{abstract}
Epilepsy is a common neurological disorder in which the normal activity of the brain cells is sometimes disturbed this can result in strange sensation, emotions and behaviors. The aim of the work is to study neurobehavioral changes that occur in group of children suffering from idiopathic epilepsy. This retrospective study included 80 children and adolescents, 50 of them with age range 2-6 years (28 males and 22 females, age $42.32 \pm 10.949$ months) and 30 with age range 6-18 years (17 males and 13 females, age $9.57 \pm 2.369$ years), enrolled from outpatient clinic of pediatric psychiatry\& neurology Benha University Hospital.All patients were subjected to a complete history taking with stress on age of onset, duration of attack, character of Keywords: Epilepsy, cognitive and psychiatric disorders neurobehavioral, internalizing, externalizing
\end{abstract}

\section{Introduction}

Epilepsy is a chronic disorder characterized by recurrent unprovoked seizures of cerebral origin with motor, sensory or autonomic disturbance with or without loss of consciousness (Zarczuk, 2010). Epileptic seizures are the clinical manifestations including symptoms and signs of an abnormal, excessive, and hypersynchronous electrical discharge of the neurons in the brain (AbouKhalil, 2008).

Seizure or convulsion is a paroxysmal, time limited change in motor activity and-or behavior that results from an abnormal electrical activity in the brain. attack. Protocol of management response to treatment \& follow up after treatment. Complete clinical examination including complete neurological examination associated with systemic examination. The child behavioral checklist Questionnaire was conducted on all the children.The results showed that there was no statistically significant difference between both groups regarding gender, type of epilepsy and regularity of treatment. There was statistically significant higher clinical internalizing withdrawn scale in group (1) in patients with 2-6 years compare to group (2) $(\mathrm{p}=0.000)$ and statistically significant higher borderline and clinical externalizing scales in group (2) in patients with 6-18 years compare to group (1).

Most seizures in children are provoked by somatic disorder originating outside the brain, such as high fever, infection, syncope, head trauma, hypoxia, toxin, or cardiac arrhythmia (Johnston, 2010). It is considered to be a heterogeneous condition, resulting from various causes and consisting of different syndromes and different seizure types.

Idiopathic epilepsy is likely to be an epilepsy without evident cause, no neurological disorders, free MRI, CT (Michael V.Johnston;2004).n About 50 million people worldwide have epilepsy with almost $80 \%$ of these people being in developing countries [WHO, 2001]. Epilepsy is more likely to occur in young children or people over the age of 65 years; however it can occur at any time (The National Society for Epilepsy, 2009). 
Epilepsy can be complicated by neurobehavioral co morbidities, which include cognitive impairment, psychiatric disorders, and social problems. Neurological comorbiditie occur very frequently in children with epilepsy. It's include learning disabilities and developmental delay, which occur in 40-50\% of children with epilepsy (Beca CB, et al.,2011), (Russ SA, et al.,2012). Nevertheless, individuals with autism and epilepsy have poorer cognitive (lower IQ), adaptive, behavioral, and social outcomes than those with autism without epilepsy, and epilepsy accounts for increased morbidity and mortality in individuals with autism (Gillberg et al., 2010).

Autism is a neurodevelopmental disorder affecting primarily social cognition but associated with language impairments, restricted interests, and repetitive behaviors. The term autism spectrum disorders (ASDs) is used interchangeably with autism and includes a broader group of children including those with autistic disorder, pervasive developmental disorders not otherwise specified, and Asperger syndrome (Korczyn et al., 2013).

Unlike for the conduct disorder variant, there is an impairment criterion for the oppositional defiant type: the symptoms must be maladaptive and inconsistent with the child or young person's developmental level. Where there are sufficient symptoms of a co morbid disorder to meet diagnostic criteria, ICD-10 discourages the application of a second diagnosis, and instead offers a single, combined category for the most common combinations (Timmer et al., 2010).

Findings from epidemiological investigations unequivocally demonstrate an increased prevalence of epilepsy (Lin et al., 2012). In addition, epidemiological studies conducted in the last thirty years have shown that behavioral disorders in children with epilepsy are 4.8 times greater than children from the general population (Plioplys et al., 2007). Findings of recent studies in children with new onset idiopathic epilepsy showed higher rates of psychopathology than healthy children (Jones et al., 2007).

The most frequently reported problems to be depression, anxiety, poor quality of life (Ottoman et al., 2011). Association between attention deficit hyperactivity disorder and childhood epilepsy has also been demonstrated (Rappley, 2005).The present work was aimed to study neurobehavioral changes that occur in group of children suffering from idiopathic epilepsy.

\section{Patients and Methods}

The following study included 80 children and adolescents who visited the outpatient clinic of Benha university hospital and who agreed to participate in the current study Collection of the studied sample took place from August 2014 to June 2015. The cases were divided as follows 50 of them with ages ranging from 2 to 6 years old (28 males and 22 females) and 30 of them with ages ranging from 6 to 18 years old (17 males and 13 females).

Exclusion criteria were

-Children younger than 2 years and elder than 18 years. -Child with a chronic illness (eg. asthma, diabetes, cardiac disease, or migraine).
-Children not diagnosed as idiopathic epilepsy (2ry epilepsy, organic brain lesion).

-Children with Mental retardation or sensory loss.

All children and parents were subjected to:-

1.Full medical history (age, sex, education, socioeconomic state, milestones. Detailed medical history about age of onset of the epilepsy, character of the attack, recurrence and duration of the seizure, medications, protocol of management, response, regularity, causes of irregularity, side effects whether controlled or not and follow up

2.Complete clinical examination including :General and systemic examination: head and neck, extremities, chest, heart and abdomen

3.Complete neurological examination: skull, cranial nerves, motor system (power and tone), sensory, reflexes and clonus.

4.EEG was done under or had already been with the patient. 5.Assessment of the behavioral status using:-

CBCL for age 2-6 years old.

CBCL for age 6-18 years old

The Child Behavior Checklist (CBCL) is a widely used instrument that assesses behavioral problems. It is a group of questions orally administered to a parent, who rates the presence and frequency of certain behaviors on a 3-point scale ( $0=$ not true, $1=$ somewhat or sometimes true, and 2 $=$ very true or often true). All items are written and take about 15 minutes to complete (Achenbach, 2001).An oral consent was signed by parents before the commencement of the study. Obtained results were statistically analyzed through mean, SE, T-test, Chi-square and ANOVA using SPSS V17 software computer package.

\section{Results}

Table1 shows the descriptive data of the study group with age range 2-6 years, their age ranged between 24 and 60 months with mean of $42.32 \pm 10.949$ months. The age of onset of seizers ranged between 2 and 48 months with mean of $18.980 \pm 12.352$ months. The relationship between the child and the caregiver was mother in 39 (78\%), father in 8 (16\%) and grandparent in 3 (6\%).

Regarding type of epiliepsy in the study group with age range 2-6 years, 12 (24\%) had simple partial, 11 (22\%) had absence seizures, 8 (16\%) had colonic seizures, $8(16 \%)$ had myoclonic seizures, 7 (14\%) had tonic seizures and $4(8 \%)$ had idiopathic tonic colonic seizures (table2).Table 3 shows non statistically significant difference between both groups regarding gender $(\mathrm{p}=0.570)$.

The percentage of normal, borderline and clinical scors of child behavior chicklistin group with age 2-6 years is depicated in table 4 . There was higher total internalizing clinical score (66\%), withdrawn problems (52\%), affective problems (38\%), ADHD (36\%) and ODD (36\%).

Table 5 shows the percentage of normal, borderline and clinical scores of child behavior checklistin group with age 6-18 years. There was higher total internalizing clinical score $(53.3 \%)$, other problems $(46.7 \%)$ and DSM-scales affective problems (23.3\%). 
Table (1): Descriptive data of group with age ranging from 2-6 years

\begin{tabular}{|cc|c|c|c|c|}
\hline \multicolumn{2}{|c|}{$\mathbf{n = 5 0}$} & Min & Max & Mean & SD \\
\hline \multicolumn{2}{|c|}{$\begin{array}{c}\text { Age (m) } \\
\text { Age of onset (m) }\end{array}$} & 24.00 & 60.00 & 42.320 & 10.949 \\
& & \multicolumn{2}{|c|}{ Frequency } & Percent \\
\hline & Male & \multicolumn{2}{|c|}{28} & 56.0 \\
\hline Sex & Female & 22 & 44.0 \\
\hline $\begin{array}{c}\text { Accompanied person } \\
\text { to child }\end{array}$ & Mother & 39 & 78.0 \\
& Father & 8 & 16.0 \\
& Grandparent & 3 & 6.0 \\
\hline
\end{tabular}

Table (2): Descriptive data of group with age ranging from6-18 years

\begin{tabular}{|c|c|c|c|c|c|}
\hline \multicolumn{2}{|l|}{$n=30$} & Min & Max & Mean & SD \\
\hline \multirow{2}{*}{\multicolumn{2}{|c|}{$\begin{array}{c}\text { Age (ys) } \\
\text { Age of onset (ys) }\end{array}$}} & 6.50 & 15.00 & 9.570 & 2.369 \\
\hline & & 1.00 & 8.00 & 4.133 & 1.833 \\
\hline & & \multicolumn{2}{|c|}{ Frequency } & \multicolumn{2}{|c|}{ Percent } \\
\hline \multirow[t]{2}{*}{ Sex } & Male & \multicolumn{2}{|c|}{17} & \multicolumn{2}{|c|}{56.7} \\
\hline & Female & \multicolumn{2}{|c|}{13} & \multicolumn{2}{|c|}{43.3} \\
\hline \multirow{3}{*}{$\begin{array}{c}\text { Accompanied person } \\
\text { to child }\end{array}$} & Mother & \multicolumn{2}{|c|}{22} & \multicolumn{2}{|c|}{73.3} \\
\hline & Father & \multicolumn{2}{|c|}{7} & \multicolumn{2}{|c|}{23.3} \\
\hline & Grandparent & \multicolumn{2}{|c|}{1} & \multicolumn{2}{|c|}{3.3} \\
\hline
\end{tabular}

Table (3): Gender difference between both groups

\begin{tabular}{|c|c|c|c|c|c|c|c|}
\hline & & & $\begin{array}{c}\text { Group (1) } \\
\text { between 2-6 } \\
\text { ys }\end{array}$ & $\begin{array}{c}\text { Group (2) } \\
\text { between 6-18 } \\
\text { ys }\end{array}$ & Total & X & $\mathrm{P}$ \\
\hline \multirow[t]{4}{*}{ Gender } & Male & $n^{\circ}$ & 28 & 17 & 45 & \multirow{4}{*}{0.954} & \multirow{4}{*}{0.570} \\
\hline & & $\%$ & $56.0 \%$ & $56.7 \%$ & $56.2 \%$ & & \\
\hline & \multirow[t]{2}{*}{ Female } & $n^{\circ}$ & 22 & 13 & 35 & & \\
\hline & & $\%$ & $44.0 \%$ & $43.3 \%$ & $43.8 \%$ & & \\
\hline \multirow{2}{*}{\multicolumn{2}{|c|}{ Total }} & $n^{\circ}$ & 50 & 30 & 80 & & \\
\hline & & $\%$ & $100.0 \%$ & $100.0 \%$ & $100.0 \%$ & & \\
\hline
\end{tabular}


Table (4): Child behavior checklist in studygroup with age ranging from 2-6 years

\begin{tabular}{|cc|c|c|c||}
\hline & & Normal & Borderline & Clinical \\
\cline { 3 - 5 } & & $\mathrm{n}(\%)$ & $\mathrm{n}(\%)$ & $\mathrm{n}(\%)$ \\
\hline \hline Internalizing & Emotionally & $31(62)$ & $14(28)$ & $5(10)$ \\
& Anxious depression & $30(60)$ & $12(24)$ & $8(16)$ \\
& Somatic complaints & $17(34)$ & $22(44)$ & $11(22)$ \\
& Withdrawn & $20(40)$ & $4(8)$ & $26(52)$ \\
& Total & $7(14)$ & $10(20)$ & $33(66)$ \\
\hline \hline Sleep problems & & $32(64)$ & $11(22)$ & $7(14)$ \\
\hline \hline Externalizing & Attention & $37(74)$ & $6(12)$ & $7(14)$ \\
& Aggression & $47(94)$ & $3(6)$ & 0 \\
& Total & $44(88)$ & $5(10)$ & $1(2)$ \\
\hline \hline Other problems & & $33(66)$ & $6(12)$ & $11(22)$ \\
\hline \hline Oriented scale & Affective problems & $14(28)$ & $17(34)$ & $19(38)$ \\
& Anxiety problems & $26(52)$ & $19(38)$ & $5(10)$ \\
& PDD & $20(40)$ & $12(24)$ & $18(36)$ \\
& ADHD & $26(52)$ & $6(12)$ & $18(36)$ \\
& ODD & $50(100)$ & 0 & 0 \\
\hline \hline Language development survey & $50(100)$ & 0 & 0 \\
\hline \hline
\end{tabular}

Table 6 shows non statistically significant difference between both groups regarding type of epilepsy $(p=0.577)$. Table 7 shows Difference in regularity of treatment in study groups and there is no statistically significant difference between both groups regarding regularity of treatment $(\mathrm{p}=0.408)$ (table 8$)$. Also there is no statistically significant difference between both groups regarding internalizing scale $(\mathrm{p}=0.467)$ (table 9). Table 10 shows higher statistically significant clinical internalizing withdrawn scale in group (1) in patients with 2-6 years compare to group (2) $(\mathrm{p}=0.000)$. Non statistically significant difference was recorded between both groups regarding externalizing aggression scale $(\mathrm{p}=0.402)$ (table 11). Table 12 shows difference in other problem scale in study groups.

In conclusion,the results showed that there was non statistically difference between both groups regarding gender, type of epilepsy and regularity of treatment. There was statistically significant higher clinical internalizing withdrawn scale in group (1) in patients with 2-6 years compare to group $(2)(p=0.000)$ and statistically significant higher borderline and clinical externalizing and other problem scales in group (2) in patients with 6-18 years compare to group (1).

\section{Discussion}

Epilepsy is a chronic neurologic disorder characterized by unprovoked recurrent seizures. Meguins, et al.,(2015) believed it to be the most comon chronic neurological disorder affecting 0.4 to $1 \%$ of the greneral population. Children with epilepsy have elevated rates of behavioral problems.It has been established that children with epilepsy have behavior problems at rates almost 5 times higher than those of the general population children(Davis et al.,2003). Studies investigating children with seizures show that behavior problems occur early in the course of the disorder and, in some children, even precede seizure onset ( Jones et al., 2007). 
Table (5): CHILD BEHAVIOR CHECKLIST in study group with age ranging from 6-18 years

\begin{tabular}{|rr|c|c|c|}
\hline & & Normal & Borderline & Clinical \\
\cline { 3 - 5 } & & $\mathrm{n}(\%)$ & $\mathrm{n}(\%)$ & $\mathrm{n}(\%)$ \\
\hline \hline Internalizing & Anxious depression & $22(73.3)$ & $8(26.7)$ & 0 \\
& Somatic complaints & $30(100)$ & 0 & 0 \\
Withdrawn & $7(23.3)$ & $15(50)$ & $8(26.7)$ \\
Total & $7(23.3)$ & $7(23.3)$ & $16(53.3)$ \\
\hline \hline Social problems & $22(73.3)$ & $3(10)$ & $5(16.7)$ \\
\hline \hline Thought problems & $22(73.3)$ & $8(26.7)$ & 0 \\
\hline \hline Extention problems & $26(86.7)$ & $4(13.3)$ & 0 \\
\hline \hline Othernalizing problems & Role breaking & $27(90)$ & $2(6.7)$ & $1(3.3)$ \\
& Aggressive behavior & $27(90)$ & $3(10)$ & 0 \\
Total & $13(48.3)$ & $11(36.7)$ & $6(20)$ \\
\hline \hline DSM scales & Affective problems & $11(36.7)$ & $12(40)$ & $7(23.3)$ \\
& Anxiety problems & $24(80)$ & $3(10)$ & $3(10)$ \\
& Somatic problems & $29(96.7)$ & $1(3.3)$ & 0 \\
& ADHD & $27(90)$ & $3(10)$ & 0 \\
& ODD & $24(80)$ & $5(16.7)$ & $1(3.3)$ \\
& CD & $28(93.3)$ & $2(6.7)$ & 0 \\
\hline \hline Competence & Sluggish & $26(86.7)$ & $4(13.3)$ & 0 \\
& Social & $28(93.3)$ & $2(6.7)$ & 0 \\
& School & $17(56.7)$ & $8(26.7)$ & $5(16.7)$ \\
\hline \hline
\end{tabular}

This retrospective study included 80 children and reported that epilepsy affects $0.5-1 \%$ of children younger adolescents, 50 of them with age ranging from 2-6 years than 16 years.

(28 males and 22 females, age $42.32 \pm 10.949$ months) and 30 with age ranging from 6-18 years (17 males and 13 females, age 9.57 \pm 2.369 years), enrolled from outpatient clinic of pediatric psychiatry and neurology Banha University Hospital.The aim of the work is to study neurobehavioral changes that occur in children with idiopathic epilepsy. Results of the current study show that the age of the onset of seizure in group 1 that age ranging from 2 to 6 years with mean of $18.980 \pm 12.352$ months. The age of onset of seizers in group 2 ranged between 1 and 8
years with mean of $4.13 \pm 1.83$ years. This comes in agreement with Jones and his collegues (2008) who
Results of the current study shows that there was non statistically significant difference between both groups regarding gender $(\mathrm{p}=0.570)$. Although Mitaki (2011) states that males have slightly higher risk than females for developing epilepsy. Austin et al., (2007), found that neither sex of child nor child age was significantly associated with total behavior problems at any time. These findings, however, are consistent with most studies, which have not shown a relationship between age at onset and behavior problems. 
Table (6): Difference in type of epilepsy in study groups

\begin{tabular}{|c|c|c|c|c|c|c|c|}
\hline & & & $\begin{array}{c}\text { Group (1) } \\
\text { between 2- } \\
6 \text { ys }\end{array}$ & $\begin{array}{c}\text { Group (2) } \\
\text { between } \\
6-18 \text { ys }\end{array}$ & Total & $\mathrm{x}$ & $\mathrm{p}$ \\
\hline \multirow[t]{6}{*}{$\begin{array}{l}\text { Type of } \\
\text { epilepsy }\end{array}$} & Idiopathic tonic & $\begin{array}{l}\mathbf{n}^{\circ} \\
\%\end{array}$ & $\begin{array}{c}7 \\
14.0 \%\end{array}$ & $\begin{array}{c}4 \\
13.3 \%\end{array}$ & $\begin{array}{c}11 \\
13.8 \%\end{array}$ & \multirow{6}{*}{3.810} & \multirow{6}{*}{0.577} \\
\hline & $\begin{array}{l}\text { Idiopathic } \\
\text { colonic }\end{array}$ & $\begin{array}{l}\mathbf{n}^{\circ} \\
\%\end{array}$ & $\begin{array}{c}8 \\
16.0 \%\end{array}$ & $\begin{array}{c}8 \\
26.7 \%\end{array}$ & $\begin{array}{c}16 \\
20.0 \%\end{array}$ & & \\
\hline & $\begin{array}{l}\text { Idiopathic tonic } \\
\text { colonic }\end{array}$ & $\begin{array}{l}\mathbf{n}^{\circ} \\
\%\end{array}$ & $\begin{array}{c}4 \\
8.0 \%\end{array}$ & $\begin{array}{c}1 \\
3.3 \%\end{array}$ & $\begin{array}{c}5 \\
6.2 \%\end{array}$ & & \\
\hline & $\begin{array}{l}\text { Idiopathic } \\
\text { Absence }\end{array}$ & $\begin{array}{l}\mathbf{n}^{\circ} \\
\%\end{array}$ & $\begin{array}{c}11 \\
22.0 \%\end{array}$ & $\begin{array}{c}3 \\
10.0 \%\end{array}$ & $\begin{array}{c}14 \\
17.5 \%\end{array}$ & & \\
\hline & $\begin{array}{c}\text { Idipathic simple } \\
\text { partial }\end{array}$ & $\begin{array}{l}\mathbf{n}^{\circ} \\
\%\end{array}$ & $\begin{array}{c}12 \\
24.0 \%\end{array}$ & $\begin{array}{c}7 \\
23.3 \%\end{array}$ & $\begin{array}{c}19 \\
23.8 \%\end{array}$ & & \\
\hline & $\begin{array}{l}\text { Idiopathic } \\
\text { myoclonic }\end{array}$ & $\begin{array}{l}\mathbf{n}^{\circ} \\
\%\end{array}$ & $\begin{array}{c}8 \\
16.0 \%\end{array}$ & $\begin{array}{c}7 \\
23.3 \%\end{array}$ & $\begin{array}{c}15 \\
18.8 \%\end{array}$ & & \\
\hline \multicolumn{2}{|c|}{ Total } & $\begin{array}{l}\mathbf{n}^{\circ} \\
\%\end{array}$ & $\begin{array}{c}50 \\
100.0 \%\end{array}$ & $\begin{array}{c}30 \\
100.0 \%\end{array}$ & $\begin{array}{c}80 \\
100.0 \%\end{array}$ & & \\
\hline
\end{tabular}

Table (7): Difference in regularity of treatment in study groups

\begin{tabular}{|c|c|c|c|c|c|c|c|}
\hline & & & $\begin{array}{c}\text { Group (1) } \\
\text { between } \\
2-6 \text { ys }\end{array}$ & $\begin{array}{c}\text { Group (2) } \\
\text { between } \\
6-18 \text { ys }\end{array}$ & Total & $X$ & $\mathrm{P}$ \\
\hline \multirow{4}{*}{$\begin{array}{c}\text { Regularity of } \\
\text { treatment }\end{array}$} & Regular & $n^{\circ}$ & 29 & 19 & 48 & \multirow{4}{*}{0.222} & \multirow{4}{*}{0.408} \\
\hline & & $\%$ & $58.0 \%$ & $63.3 \%$ & $60.0 \%$ & & \\
\hline & \multirow[t]{2}{*}{ Irregular } & $n^{\circ}$ & 21 & 11 & 32 & & \\
\hline & & $\%$ & $42.0 \%$ & $36.7 \%$ & $40.0 \%$ & & \\
\hline \multirow{2}{*}{\multicolumn{2}{|c|}{ Total }} & $n^{\circ}$ & 50 & 30 & 80 & & \\
\hline & & $\%$ & $100.0 \%$ & $100.0 \%$ & $100.0 \%$ & & \\
\hline
\end{tabular}


Table (8): Difference in internalizing scale in study groups

\begin{tabular}{|c|c|c|c|c|c|c|c|}
\hline & & & $\begin{array}{c}\text { Group (1) } \\
\text { between 2-6 } \\
\text { ys }\end{array}$ & $\begin{array}{c}\text { Group (2) } \\
\text { between 6- } \\
18 \text { ys }\end{array}$ & Total & $X$ & $\mathrm{P}$ \\
\hline \multirow[t]{3}{*}{ Internalizing } & Normal & $\begin{array}{l}\mathbf{n}^{\circ} \\
\%\end{array}$ & $\begin{array}{c}7 \\
14.0 \%\end{array}$ & $\begin{array}{c}7 \\
23.3 \%\end{array}$ & $\begin{array}{c}14 \\
17.5 \%\end{array}$ & \multirow{3}{*}{1.523} & \multirow{3}{*}{0.467} \\
\hline & Borderline & $\begin{array}{l}\mathbf{n}^{\circ} \\
\%\end{array}$ & $\begin{array}{c}10 \\
20.0 \%\end{array}$ & $\begin{array}{c}7 \\
23.3 \%\end{array}$ & $\begin{array}{c}17 \\
21.2 \%\end{array}$ & & \\
\hline & Clinical & $\begin{array}{l}\mathbf{n}^{\circ} \\
\%\end{array}$ & $\begin{array}{c}33 \\
66.0 \%\end{array}$ & $\begin{array}{c}16 \\
53.3 \%\end{array}$ & $\begin{array}{c}49 \\
61.2 \%\end{array}$ & & \\
\hline \multicolumn{2}{|c|}{ Total } & $\begin{array}{l}\mathbf{n}^{\circ} \\
\%\end{array}$ & $\begin{array}{c}50 \\
100.0 \%\end{array}$ & $\begin{array}{c}30 \\
100.0 \%\end{array}$ & $\begin{array}{c}80 \\
100.0 \%\end{array}$ & & \\
\hline
\end{tabular}

Table (9): Difference in internalizing withdrawn scale in study groups

\begin{tabular}{|c|c|c|c|c|c|c|c|}
\hline & & & $\begin{array}{c}\text { Group (1) } \\
\text { between 2-6 } \\
\text { ys }\end{array}$ & $\begin{array}{c}\text { Group (2) } \\
\text { between 6- } \\
18 \text { ys }\end{array}$ & Total & $X$ & $P$ \\
\hline \multirow[t]{3}{*}{$\begin{array}{c}\text { Internalizing } \\
\text { withdrawn }\end{array}$} & Normal & $\begin{array}{l}\mathbf{n}^{\circ} \\
\%\end{array}$ & $\begin{array}{c}20 \\
40.0 \%\end{array}$ & $\begin{array}{c}7 \\
23.3 \% \\
\end{array}$ & $\begin{array}{c}27 \\
33.8 \% \\
\end{array}$ & \multirow{3}{*}{18.301} & \multirow{3}{*}{$\begin{array}{l}0.000 \\
\text { (HS) }\end{array}$} \\
\hline & Borderline & $\begin{array}{l}\mathbf{n}^{\circ} \\
\%\end{array}$ & $\begin{array}{c}4 \\
8.0 \%\end{array}$ & $\begin{array}{c}15 \\
50.0 \% \\
\end{array}$ & $\begin{array}{c}19 \\
23.8 \%\end{array}$ & & \\
\hline & Clinical & $\begin{array}{l}\mathbf{n}^{\circ} \\
\%\end{array}$ & $\begin{array}{c}26 \\
52.0 \%\end{array}$ & $\begin{array}{c}8 \\
26.7 \% \\
\end{array}$ & $\begin{array}{c}34 \\
42.5 \%\end{array}$ & & \\
\hline \multicolumn{2}{|c|}{ Total } & $\begin{array}{l}\mathbf{n}^{\circ} \\
\%\end{array}$ & $\begin{array}{c}50 \\
100.0 \%\end{array}$ & $\begin{array}{c}30 \\
100.0 \%\end{array}$ & $\begin{array}{c}80 \\
100.0 \%\end{array}$ & & \\
\hline
\end{tabular}

Recurrent un-controlled seizures significantly years; there was higher total internalizing clinical score predicted behavior problems is very early in the course of (66\%), withdrawn problems (52\%), affective problems seizure condition, even when key child, demographic, (38\%), ADHD (36\%) and ODD (36\%).Regarding the and seizure variables were controlled. Explanations for percentage of normal, borderline and clinical scores of these findings include the possibilities that both seizures child behavior checklist in group 2 with age 6-18 years; and behavior problems are caused by an underlying there was higher total internalizing clinical score (53.3\%), neurological disorder, that seizures per se disrupt behavior, other problems (46.7\%) and DSM-scales affective or that children have negative psychological responses problems (23.3\%). Similar to our findings, Eom et al., to seizure activity (Austin et al., 2002). Although this was (2014) found that younger age pateints showed a strong not the case in the current study with both groups regarding positive impact on psychosocial function in pediatric the regularity of the treatment. Results of our study show epilepsy, particularly for adaptive function and competence. that the percentage of normal, borderline and clinical scores of child behavior checklist in group 1 with age 2-6 
Table (10): Difference in externalizing scale in study groups

\begin{tabular}{|c|c|c|c|c|c|c|c|}
\hline & & & $\begin{array}{c}\text { Group (1) } \\
\text { between } \\
2-6 \text { ys }\end{array}$ & $\begin{array}{c}\text { Group (2) } \\
\text { between } \\
6-18 \text { ys }\end{array}$ & Total & $\mathrm{x}$ & $\mathrm{P}$ \\
\hline \multirow[t]{6}{*}{ Externalizing } & Normal & $n^{\circ}$ & 44 & 13 & 57 & \multirow{6}{*}{18.961} & \multirow{6}{*}{$\begin{array}{c}0.000 \\
\text { (HS) }\end{array}$} \\
\hline & & $\%$ & $88.0 \%$ & $43.3 \%$ & $71.2 \%$ & & \\
\hline & \multirow[t]{2}{*}{ Borderline } & $\mathbf{n}^{\circ}$ & 5 & 11 & 16 & & \\
\hline & & $\%$ & $10.0 \%$ & $36.7 \%$ & $20.0 \%$ & & \\
\hline & \multirow[t]{2}{*}{ Clinical } & $\mathbf{n}^{\circ}$ & 1 & 6 & 7 & & \\
\hline & & $\%$ & $2.0 \%$ & $20.0 \%$ & $8.7 \%$ & & \\
\hline \multirow{2}{*}{\multicolumn{2}{|c|}{ Total }} & $\mathbf{n}^{\circ}$ & 50 & 30 & 80 & & \\
\hline & & $\%$ & $100.0 \%$ & $100.0 \%$ & $100.0 \%$ & & \\
\hline
\end{tabular}

Table (11): Difference in externalizing aggression scale in study groups

\begin{tabular}{|ccc|c|c|c|c|c|}
\hline & & & $\begin{array}{c}\text { Group (1) } \\
\text { between } \\
\text { 2-6 ys }\end{array}$ & $\begin{array}{c}\text { Group (2) } \\
\text { between } \\
\mathbf{6 - 1 8} \text { ys }\end{array}$ & Total & X & P \\
\hline $\begin{array}{c}\text { Externalizing } \\
\text { aggression }\end{array}$ & Normal & $\mathbf{n}^{\circ}$ & 47 & 27 & 74 & & \\
\cline { 2 - 7 } & $\%$ & $94.0 \%$ & $90.0 \%$ & $92.5 \%$ & \multirow{2}{*}{0.432} & \multirow{2}{*}{0.402} \\
\cline { 2 - 7 } & Borderline & $\mathbf{n}^{\circ}$ & 3 & 3 & 6 & & \\
\hline \multicolumn{2}{|c|}{ Total } & $\%$ & $6.0 \%$ & $10.0 \%$ & $7.5 \%$ & & \\
\hline
\end{tabular}

Table (12): Difference in other problem scale in study groups

\begin{tabular}{|c|c|c|c|c|c|c|c|}
\hline & & & $\begin{array}{c}\text { Group (1) } \\
\text { between } \\
2-6 \text { ys }\end{array}$ & $\begin{array}{c}\text { Group (2) } \\
\text { between } \\
6-18 \text { ys }\end{array}$ & Total & $X$ & $\mathrm{P}$ \\
\hline \multirow[t]{6}{*}{ Other problems } & Normal & $\mathbf{n}^{\circ}$ & 33 & 11 & 44 & \multirow{6}{*}{6.881} & \multirow{6}{*}{$\begin{array}{c}0.320 \\
(\mathrm{~S})\end{array}$} \\
\hline & & $\%$ & $66.0 \%$ & $36.7 \%$ & $55.0 \%$ & & \\
\hline & \multirow[t]{2}{*}{ Borderline } & $n^{\circ}$ & 6 & 5 & 11 & & \\
\hline & & $\%$ & $12.0 \%$ & $16.7 \%$ & $13.8 \%$ & & \\
\hline & \multirow[t]{2}{*}{ Clinical } & $n^{\circ}$ & 11 & 14 & 25 & & \\
\hline & & $\%$ & $22.0 \%$ & $46.7 \%$ & $31.2 \%$ & & \\
\hline \multirow{2}{*}{\multicolumn{2}{|c|}{ Total }} & $n^{\circ}$ & 50 & 30 & 80 & & \\
\hline & & $\%$ & $100.0 \%$ & $100.0 \%$ & $100.0 \%$ & & \\
\hline
\end{tabular}




\section{References}

Abou-Khalil B.(2008). Levetiracetam in the treatment of epilepsy. Neuropsychiatr Dis Treat;4(3):50723.

Achenbach TM.(1991). Manual for the Child Behavior Checklist/4-18. Burlington, VT: University of Vermont Department of Psychiatry.

Austin JK, Caplan R. (2007). Behavioral and psychiatric comorbidities in pediatric epilepsy: toward an integrative model. Epilepsia;48:1639-51

Austin JK, Dunn DW, Caffrey HM, Perkins SM, Harezlak J, Rose DF.(2002). Recurrent seizures and behavior problems in children with first recognized seizures: a prospective study. Epilepsia;43:1564-73.

Bailet LL, Turk WR. (2000).The impact of childhood epilepsy on neurocognitive and behavioral performance: a prospective longitudinal study. Epilepsia;41(4):426-431.

Beca CB, Vickrey BG, Caplan R, (2011). Psychiatric, comorbidity and quality of life outcomes in childhood. Pediatrics.;128(6):e1532-1543.

Beghi M, Cornaggia CM, Frigeni B, Beghi E.(2006). Learning disorders in epilepsy. Epilepsia.;47(suppl 2):1418.

Caplan R, Siddarth P, Gurbani S, Hanson R, Sankar R, Shields WD.(2005). Depression and anxiety disorders in pediatric epilepsy. Epilepsia.;46(5):720-730.

Caplan R, Wu KN, Lieber E, Siddarth P, Smith K, Sankar R. (2008).Dealing with epilepsy: parents speak up. Epilepsy Behav.;13(1):131-138

Chabolla DR (2002). Characteristics of the epilepsies. Mayo Clin Proc.;77(9):981-90.

Davies S, Heyman I, Goodman R. (2003). A population survey of mental health problems in children with epilepsy. Dev Med Child Neurol;45:292-5.

Dunn DW, Austin JK, Harezlak J, Ambrosius WT.(2003). ADHD and epilepsy in childhood. Dev Med Child Neurol.;45(1): 50 - 54.

Eom S1, Eun SH2, Kang HC3, Eun BL2, Nam SO4, Kim SJ5, Chung HJ6, Kwon SH7, Lee YM8, Lee JS3, Kim DW9, Oh KJ10, Kim HD (2014). Epilepsy-related clinical factors and psychosocial functions in pediatric epilepsy.Epilepsy Behav.;37:43-8.

Erikson, K.J. and Koivikko M.J. (2009). prevalence, classification and severity of epileptic syndromes in children; 38 (12): 1275- 1282

Fisher RS, Harding G, Erba G, Barkley GL, Wilkins A. (2005). Epilepsy Foundation of America Working Group. Photic- and pattern-induced seizures: a review for the EpilepsyEpilepsia.;46(9):1426-41.

Gaby AR.(2007). Natural approaches to epilepsy. Altern Med Rev;12(1):9-24.

Gillberg C, Billstedt E, Sundh V, Gillberg IC.(2010). Mortality in autism: a prospective longitudinal community- 872 . based study. J Autism DevDisord;40:352-7.
ILAE Commission Report, (b) (2010). The epidemiology of the epilepsies: future directions: International League Against Epilepsy. Epilepsia; 44:17-18.

ILAE, Commission Report, (a) (2010).History and Segmata of epil Achenbach TM. Manual for the Child Behavior Checklist/4-18. Burlington, VT:University of Vermont Department of Psychiatry; 1991 epsy: future directions: International League Against Epilepsy. Epilepsia;. 44:12-14.

Johnston, M.V. (2010). Seizures in children. In: Nelson Text book of paediatc. Nelson W.E (editor) Sounderco. Philadelphia, LondonToranto, 17th_ed2010; 593:2457-2471

Jones JE, Austin JK, Caplan R, Dunn D, Plioplys S, Salpekar JA.(2008). Psychiatric disorders in children and adolescents who have epilepsy. Pediatr Rev.;29(2):e9-e14 Jones JE, Watson R, Sheth R.(2007). Psychiatric comorbidity in children with new onset epilepsy. Dev Med Child Neurol; 49 : 493 - 7.

Kaufmann R, Goldberg-Stern H, Shuper A.(2009). Attention-deficit disorders and epilepsy in childhood: incidence, causative relations and treatment possibilities. J Child Neurol.;24(6):727-733.

Korczyn AD1, Schachter SC, Brodie MJ, Dalal SS, Engel J Jr, Guekht A, Hecimovic H, Jerbi K, KannerAM,Johannessen JLandmark C, Mares P, Marusic P, Meletti S, Mula M, Patsalos PN, Reuber M, Ryvlin P, Štillová K, Tuchman R, Rektor I.(2013). Epilepsy, cognition, and neuropsychiatry (Epilepsy, Brain, and Mind, part 2). Epilepsy Behav.;28(2):283-302.

Lin JJ, Mulla $\mathrm{M}$ and Hermann BP (2012).Uncovering the neurobehavioral co-morbidities of epilepsy over life span. Lancet.;380(9848):1180-1192.

Meguins LC1, Adry RA1, Silva-Junior SC1, AraújoFilho GM2, MarquesLH(2015).. Shorter epilepsy duration is associated with better seizure outcome in temporal lobe epilepsy surgery.Journal of ArqNeuropsiquiatr.;73(3):212-7.

Menkes, J.H. (2005). Paroxysmal Disorders. In text book of child neurology, 6th ed. By menkes J.H. Sarnet H.B. (eds). Lippincott Williams\& Philadelphia, London, New York; 919: 1007.

Michael V.J.(2004). Seizures in childhood, Nelsonpediatrics; vol.4, $17^{\text {th }}$ edition 2004.

Mikati MA1, Daderian R, Zeinieh M, Leonard AS, Azzam D, Kurdi R,(2011). Potential neuroprotective effects of continuous topiramate therapy in the developing brain; Epilepsy Behav.;20(4):597-601.

Murphy CC, Trevathan E, Yeargin-Allsopp M. (1995).Prevalence of epilepsy and epileptic seizures in 10year-old children: results from the Metropolitan Atlanta Developmental Disabilities Study. Epilepsia.;36(9):866- 
Ottman, R., Lipton, R.B., Ettinger, A.B., Cramer, J.A., Reed, M.L., Morrison, A., \& Wan, G.J (2011). Comorbidities of epilepsy: results from the Epilepsy Comorbidities and Health (EPIC) survey. Epilepsia.;52(2):308-15.

Plioplys S, Dunn D, Caplan R. (2007).Psychiatric Problems in Children with Epilepsy: 10-year Research Review Update. Journal of the American Academy of Child and Adolescent Psychiatry.;46(11):1389-1402.

Plioplys S. (2003). Depression in children and adolescents with epilepsy. Epilepsy Behav.;4(suppl 3):S39S45

Rappley M.(2005). Attention- deficit hyperactivity disorder. N Engl J Med.;352:165-173.

Russ SA, Larson K and Halfon N. (2012). National profile of childhood epilepsy and seizure disorder. Pediatrics.;129(2):256-264.

Timmer SG, Zebell NM, Culver MA, Urquiza AJ (2010). Efficacy of adjunct in-home coaching to improve outcomes in parent-child interaction therapy. Research on Social Work Practice.;20:36-45.

Van RijckevorselK.(2006). Cognitive problems related to epilepsy syndromes, especially malignant epilepsies. Seizure.;15(4):227-34

WHO, (2008).World Health Organization. International classification of impairments, disabilities and handicaps. A manual of classification relating to the consequence of disease. Geneva.

Zarczuk R, Łukasik D, Jedrych M, BorowiczKK. (2010). Pharmacol Rep. Immunological aspects of epilepsy. 62(4):592-607. 